\title{
Magnetic suppression of secondary electrons in plasma immersion ion implantation
}

\author{
Ing Hwie Tan, ${ }^{\text {a) }}$ Mario Ueda, Renato S. Dallaqua, and Jose O. Rossi \\ Instituto Nacional de Pesquisas Espaciais, Laboratório Associado de Plasmas, Av. Dos Astronautas 1758, \\ CEP 12227-010, São Jose dos Campos, S. P. Brazil
}

(Received 2 August 2004; accepted 18 November 2004; published online 6 January 2005)

\begin{abstract}
In this work, magnetic suppression of secondary electrons in plasma immersion ion implantation is demonstrated experimentally in a vacuum arc system. Secondary electrons emitted normally to a copper sample surface were detected by a Faraday cup, whose signal exhibited large negative spikes coincident with high voltage pulses when aluminum ions of an unmagnetized plasma were implanted. When a $12.5 \mathrm{mT}$ magnetic field parallel to the sample's surface is applied, these spikes are not seen, showing that secondary electrons were magnetically suppressed. Another cup, oriented to detect electrons that flow along the field lines, does not exhibit such negative spikes in either unmagnetized or magnetized plasmas, indicating that a virtual cathode was formed by the trapped secondary electrons. (C) 2005 American Institute of Physics. [DOI: 10.1063/1.1852704]
\end{abstract}

Plasma immersion ion implantation (PIII) is a non-lineof-sight technique that eliminates the necessity of target manipulation. It consists of applying negative high voltage pulses to a target immersed in a plasma medium, from where ions are extracted directly and accelerated into the target, resulting in a homogeneous surface implantation from all sides. Several works have demonstrated the successful metallurgical and semiconductor implantation using this technique. ${ }^{1,2}$ The use of ion implantation using plasmas made of metallic ion specie ${ }^{3,4}$ has also been demonstrated to have numerous applications, when vacuum arc sources proved to be excellent devices for the production of metallic plasmas. ${ }^{5}$

Secondary electrons emitted due to high energy ion bombardment of material surfaces in PIII can decrease significantly the efficiency of this technique, since a large portion of power is lost into electron energy. Secondary electron emission coefficient can be as large as 20 in metal surfaces, reducing efficiency to values as low as $5 \%$. Furthermore, for electron energies higher than about $40 \mathrm{keV}$, electron impact with chamber walls produces hazardous x-ray radiation. ${ }^{6}$ One technique used to suppress X-ray generation is based on electrostatic confinement of secondary electrons, which are trapped within a metal enclosure biased to the same potential as the target. ${ }^{7}$ Secondary electrons are repeatedly reflected within this enclosure, and are prevented from impacting the chamber walls. Efficiency could be improved if electrons dissipate their energy into the plasma during reflections. Despite the success in reducing $\mathrm{x}$-ray levels, to our knowledge efficiency improvement was not demonstrated.

Suppression of secondary electrons has been proposed by Rej et $a l .{ }^{8}$ using an externally applied magnetic field, parallel to the target surface. Secondary electrons would be confined by the field forming an electron layer near the surface, which acts as a virtual cathode, reducing the local electric field so that subsequent emitted electrons can be reabsorbed by the surface. Since electrons are free to move in the direction parallel to the magnetic field lines, the virtual cathode will be maintained if it is formed faster than the axial transit time. If this condition is not satisfied, secondary elec-

${ }^{a)}$ Electronic mail: ingtan@plasma.inpe.br trons are emitted as two confined beams along the field lines.

Numerical simulations with low density nitrogen plasmas have been performed, showing that an electron layer would indeed be formed, ${ }^{8,9}$ but to our knowledge, this magnetic insulation method for suppressing secondary electrons in PIII has not been demonstrated experimentally.

In this work, secondary electrons emitted during ion implantation experiments in a vacuum arc aluminum plasma are directly measured by two Faraday cups with and without the presence of a magnetic field parallel to a copper target surface. Unlike most of the vacuum arc systems used for ion implantation, which have curved magnetic filters, our equipment has a straight magnetic duct and implanted surfaces are oriented parallel to the plasma stream and magnetic field, in order to avoid macroparticle contamination and minimize deposition. ${ }^{10}$ In this geometry a direct measurement of the emitted secondary electrons is possible in both the perpendicular and parallel directions to the field lines.

An aluminum cathode and a tungsten grid anode are placed inside a large and straight cylindrical vacuum chamber ( $\phi=0.22 \mathrm{~m}$ and $L=1.05 \mathrm{~m}$ ). The cathode is biased using a LC pulse-forming network with impedance matched to the plasma resistance establishing an arc current (550 A used in this experiment) with approximately $12 \mathrm{~ms}$ of flat top duration. A capacitor bank produces an axial magnetic field $B_{z}$ (12.5 $\mathrm{mT}$ used in this experiment) which is practically constant and uniform over the time and spatial scales of the experiment. A high-density plasma $\left(n \sim 10^{11} \mathrm{~cm}^{-3}\right.$ with a Gaussian profile, $T_{e} \sim 2 \mathrm{eV}$ ) is produced when the magnetic field is present, density falling by two orders of magnitude when the field is absent. ${ }^{10}$

A $20 \mathrm{~mm} \times 20 \mathrm{~mm} \times 50 \mathrm{~mm}$ copper electrode was placed inside the magnetic duct, in the uniform $B_{z}$ region, oriented with its longest dimension along $B_{z}$ and the axis of the vacuum vessel. In order to decrease the implantation currents when the magnetic field is present (which would take the high voltage pulse generator to its power limit), all faces of the electrode were isolated from the plasma with a machinable ceramic mask, except for one $20 \mathrm{~mm} \times 50 \mathrm{~mm}$ lateral face and a 10-mm-diam circular area exposed in the opposite lateral face. This copper electrode was biased from 


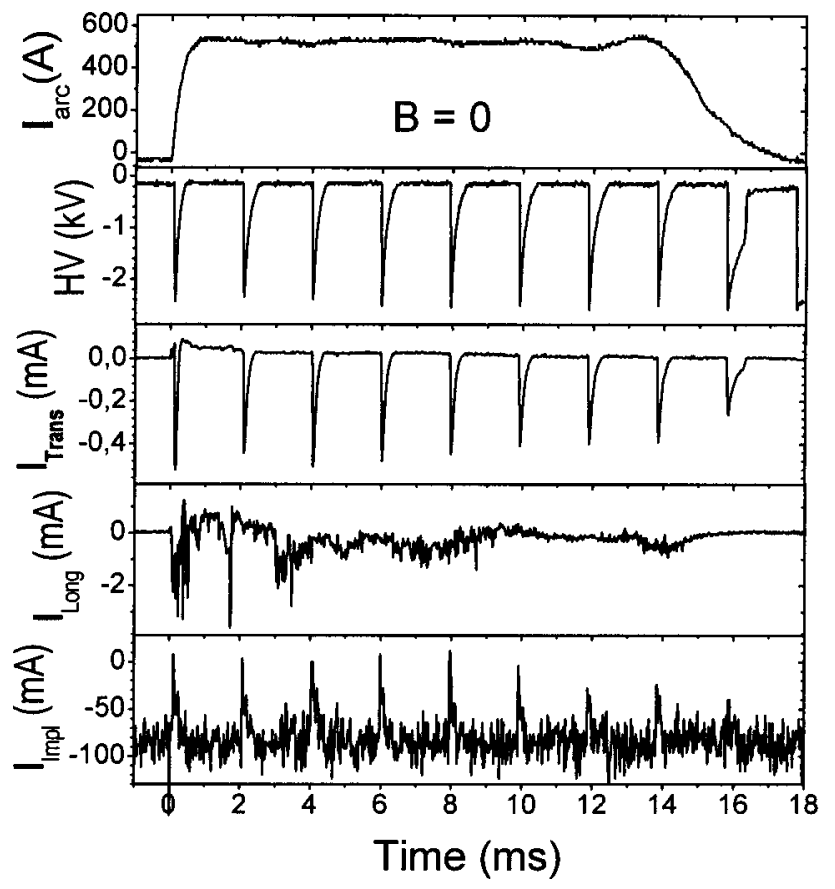

FIG. 1. Arc current, high voltage bias, transverse Faraday cup current, longitudinal cup current, and implantation current for a discharge without magnetic field.

-0.5 to $-5 \mathrm{kV}$ using a hard tube pulser, with $10 \mu$ s pulses and $500 \mathrm{~Hz}$ repetition rate.

Two Faraday cups were placed near the copper electrode to detect secondary electrons. The cups have a 24-mm-diam circular copper collector and a stainless steel suppressor grid with $30 \%$ transparency, $5 \mathrm{~mm}$ away from the collector's surface. Both the collector and the suppressor grid are mounted inside a machinable ceramic cup shielded by a grounded aluminum foil. One cup is oriented to collect electrons emerging normally to the circular exposed area. Its suppressor grid was biased to $-100 \mathrm{~V}$ and a $47 \mathrm{k} \Omega$ resistor was connected to the collector in parallel with the oscilloscope input. The other cup collects electrons that flow along the field lines parallel to the exposed $20 \mathrm{~mm} \times 50 \mathrm{~mm}$ lateral area. For this Faraday cup, biasing its grid negatively turned out to be useless when the magnetic field was present because, unlike the transversal cup which is on the plasma column's edge, this longitudinal cup was located near the center of the plasma column where the density is maximum $\left(\sim 10^{11} \mathrm{~cm}^{-3}\right)$. The Debye length in this region was estimated to be around $30 \mu \mathrm{m}$ when magnetic field is present, which is smaller than the distance between the grid's wires $(38 \mu \mathrm{m})$, so that the negative bias was shielded by the plasma. This cup was therefore operated with its grid floating, and its collector biased to $-9 \mathrm{~V}$ with a battery and connected to the oscilloscope input through a $1 \mathrm{k} \Omega$ resistor in series and $56 \Omega$ in parallel. Without magnetic field (plasma density $\sim 10^{9} \mathrm{~cm}^{-3}$ ), Debye shielding would not occur but the longitudinal cup was kept functioning as in the magnetized case for better comparison.

Figure 1 shows a typical arc discharge without magnetic field. It can be seen from the current collected by the transverse Faraday cup, that secondary electrons are emitted from the surface when energetic ions are implanted, causing large negative spikes in the cup's current coincident with the negative high voltage pulses. The longitudinal cup does not show any significant change in the collected current, indicating Downloaded 17 Jun 2005 to 150.163.34.25. Redistribution subject

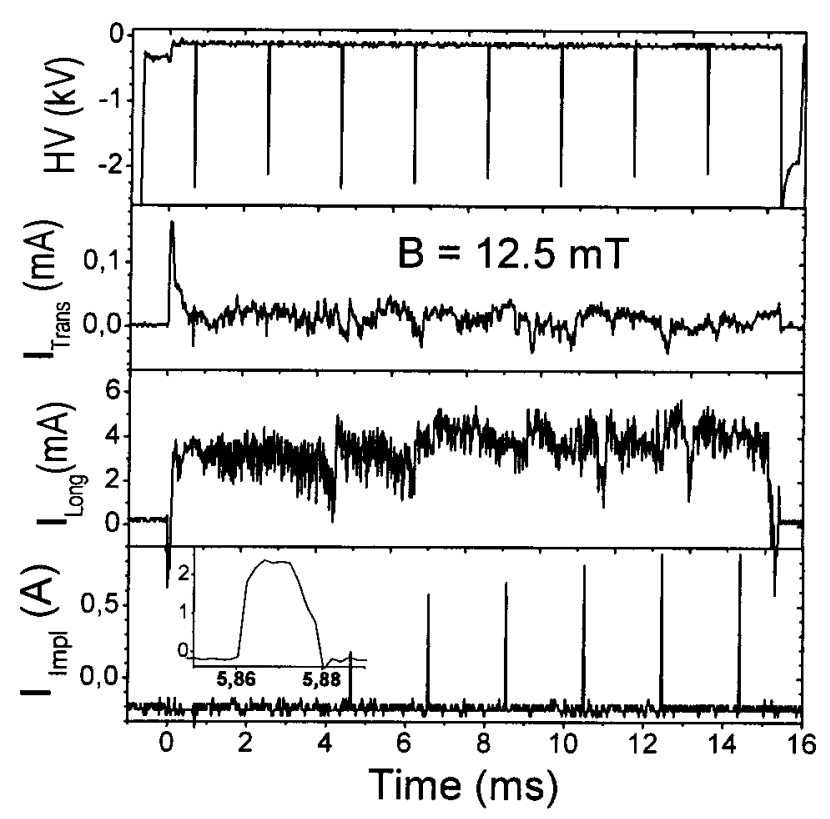

FIG. 2. High voltage bias, transverse Faraday cup current, longitudinal cup current, and implantation current for a discharge with magnetic field. The inset shows actual implantation current, which seems smaller in the expanded view due to insufficient number of samples per scope sweep in that mode.

that most of the secondaries are emitted normally to the surface even at the edge of the electrode.

When a $12.5 \mathrm{mT}$ magnetic field is applied, secondary electrons are magnetically suppressed, as can be seen in Fig. 2 . The current collected by the transversal cup does not show any negative spike as in the unmagnetized case. We can estimate the current due to secondary electrons that would be sensed by the Faraday cup if there was no suppression. From the unmagnetized case, for a high voltage of $-2.5 \mathrm{kV}$, the implantation current (measured with a Rogowsky coil) is around $80 \mathrm{~mA}$ and the amplitude of the negative spike due to secondaries is about $400 \mu \mathrm{A}$. The implantation current density is the sum of the ion current density $\left(J_{i}\right)$ and secondary electron current density $\left(J_{e}\right)$. For a singly charged ion, the secondary electron emission coefficient would then be $\gamma$ $=J_{e} / J_{i}$. Since aluminum ions in vacuum arcs have a mean charge of about ${ }^{11}+1.7$, then $\gamma=1.7 J_{e} / J_{i}$, which can be calculated considering the relevant areas and the $30 \%$ transparency of the grid, giving $\gamma=0.9$. In the magnetized case, the implantation currents at the same high voltages are around $2.5 \mathrm{~A}$, and for $\gamma=0.9$ this would imply negative spikes in the perpendicular Faraday cup current with amplitudes of 12.5 $\mathrm{mA}$, which is not observed. A detailed look at the signals does show a negative perturbation in this cup's current coincident with the high voltage pulses, but with amplitudes of the same order as the oscillations observed throughout the discharge.

Figure 2 also shows that the current in the longitudinal cup is not altered during the high voltage pulses. An oscillation of about $25 \mathrm{kHz}$ persisting throughout the discharge is attributed to drift waves, ${ }^{12}$ with no significant negative perturbation being seen beyond the amplitude of this oscillation. The numerical simulations done so far by Rej et $a l^{8}$ and Kostov et al. ${ }^{9}$ assumed low density $\left(n \sim 10^{9} \mathrm{~cm}^{-3}\right)$ plasmas with sheath thicknesses of several centimeters, in which the confined electron layer is formed a few millimeters from the to AIP license or copyright, see http://apl.aip.org/apl/copyright.jsp 
electrode's surface. Electrons in this virtual cathode can escape through the ends of the electrode along field lines. If this happens faster than the characteristic time necessary to form the electron layer, an electron beam would arise along the field lines. The fact that no significant electron current was measured by the longitudinal collector indicates that electrons diffuse away slowly enough for the virtual cathode to form. For the magnetically confined aluminum plasmas of our experiment, the plasma density is two orders of magnitude higher than the values considered in previous numerical simulations. The sheath thicknesses are much smaller than in unmagnetized cases ${ }^{13}$ and simple estimates cannot be made about the thickness and location of the electron layer. Numerical work is under way to simulate the effect of magnetic field in these high density plasmas.

In conclusion, we have demonstrated experimentally that secondary electrons emitted during ion implantation experiments in vacuum arc plasmas are suppressed by a magnetic field parallel to the sample's surface. Although our results are limited to high voltage pulses of $5 \mathrm{kV}$, this magnetic suppression method represents a simple and inexpensive way of achieving a significant gain in efficiency in PIII systems, especially at higher voltages, when secondary electron emission becomes very large.
This work was supported by FAPESP (State of Sao Paulo Research Funding Agency Process Nos. 2001/12746-0 and 2000/11114-7) and CNPq (Brazilian National Council of Scientific and Technological Development Process No. 382.208/01).

${ }^{1}$ J. R. Conrad, J. L. Radtke, R. A. Dodd, F. J. Worzala, and N. C. Tran, J. Appl. Phys. 62, 4591 (1997).

${ }^{2}$ M. Ueda, LA Berni, J. O. Rossi, J. J. Barroso, G. F. Gomes, A. F. Beloto, and E. Abramof, Surf. Coat. Technol. 136, 28 (2001)

${ }^{3}$ R. J. Adler, and S. T. Picraux, Nucl. Instrum. Methods Phys. Res. B 6, 123 (1985).

${ }^{4}$ I. G. Brown, X. Godechot, and K. M. Yu, Appl. Phys. Lett. 58, 1392 (1991).

${ }^{5}$ I. G. Brown, A. Anders, M. R. Dickinson, R. A. MacGill, and O. R. Monteiro, Surf. Coat. Technol. 112, 271 (1999).

${ }^{6}$ M. M. Shamim, J. T. Scheuer, R. P. Fetherson, and J. R. Conrad, J. Appl. Phys. 70, 4756 (1991).

${ }^{7}$ D. J. Rej, R. J. Faehl, and J. N. Matossian, Surf. Coat. Technol. 96, 45 (1997).

${ }^{8}$ D. J. Rej, B. P. Wood, R. J. Faehl, and H. H. Fleishmann, J. Vac. Sci. Technol. B 12, 861 (1994).

${ }^{9}$ K. G. Kostov, J. J. Barroso, and M Ueda, Braz. J. Phys. 34, 1689 (2004).

${ }^{10}$ I. H. Tan, M. Ueda, R. S. Dallaqua, J. O. Rossi, A. F. Beloto, E. Abramof, Y. Inoue, and O. Takai, Plasma Sources Sci. Technol. 11, 317 (2002).

${ }^{11}$ I. G. Brown, Rev. Sci. Instrum. 65, 3061 (1994).

${ }^{12}$ M. J. Hole, R. S. Dallaqua, S. W. Simpson, and E. del Bosco, Phys. Rev. E 65, 046409 (2002).

${ }^{13}$ M. Ueda, I. H. Tan, R. S. Dallaqua, J. O. Rossi, J. J. Barroso, and M. H. Tabacniks, Nucl. Instrum. Methods Phys. Res. B 206, 760 (2003). 